\title{
Devenir biologique des transuraniens inhalés $\left(^{*}\right)$
}

\author{
H. MÉTIVIER (**) \\ (Manuscrit reçu le 11 octobre 1978)
}

\begin{abstract}
RÉSUMÉ
Le devenir biologique des transuraniens inhalés est abordé par focalisations successives sur différents points d'actualité. La chimie des transuraniens conditionne grandement l'épuration pulmonaire et la translocation. Toutefois, quelques exemples montrent, en particulier pour le plutonium, que la cinétique de certaines réactions chimiques dépend de la concentration en élément; l'extrapolation aux concentrations biologiques doit donc être faite avec prudence. L'importance de l'activité spécifique est soulignée par les différences de comportement des oxydes de plutonium 238 et 239 . Elle est responsable, dans le cas de l'isotope 238 , de la dissolution rapide de l'oxyde par formation de particules nanométriques. Le rôle de ces particules, quel que soit l'isotope, est particulièrement important puisque leur devenir biologique semble bouleverser les modèles établis. Leur origine reste à établir car elles ont été identifiées aussi bien dans la combustion simultanée de $\mathrm{Pu}-\mathrm{Na}$ ou $\mathrm{Pu}-\mathrm{Mg}$ que dans la combustion de plutonium seul. Par ailleurs, l'épuration bronchique des particules microniques n'apparaît pas, pour la totalité de la fraction épurée, comme rapide. En effet, un compartiment résiduel, certes faible, séjourne relativement longtemps au niveau de l'épithélium bronchique. Cette donnée est à prendre en considération dans les calculs dosimétriques à l'échelon cellulaire. Malgré les quelques réserves apportées, la somme des connaissances acquises permet d'assez bien évaluer les risques.
\end{abstract}

\begin{abstract}
The biological behaviour of inhaled transuranic elements has been reviewed by focus on different points of present interest. Lung clearance and translocation are largely affected by the chemistry of transuranic elements. However, especially for plutonium, some examples show that the kinetics of some chemical reactions are function of the element concentration: extra-
\end{abstract}

(*) Conférence invitée lors du "Seminar on the inhalation of radionuclides 》, organisé par l'E.U.L.E.P. à Harwell, U.K., les 3 et 4 avril 1978.

(**) Commissariat à l'Énergie atomique, Institut de Protection et de Sûreté nucléaire, Département de Protection, Laboratoire de Toxicologie expérimentale, B.P. $\mathrm{n}^{\circ} 561$, 92542 Montrouge Cedex, France. 
polation to the biological concentrations must therefore be cautious. The importance of the specific activity is stressed by different behaviours of plutonium 238 and 239 oxides. In the case of ${ }^{238} \mathrm{Pu}$, it is responsible of the fast dissolution of the oxide by formation of nanometric particles. Whatever the isotopes, these particules have a particularly important function since their biological fate seems to disturb the established models. Their origin is still to be determined since they have been identified both in the combustion of $\mathrm{Pu}-\mathrm{Na}$ or $\mathrm{Pu}-\mathrm{Mg}$ simultaneously, and plutonium only. The bronchial clearance of micronic particles does not seem to be fast for the whole of the fraction cleared. A residual compartment, indeed low, will remain at the level of the bronchial epithelium for a rather long time. This must be taken into account by dosimetric evaluations at the cellular levels. In spite of the few restrictions stated, the body of knowledge makes a fair estimation of risks possible.

Depuis le premier colloque sur le diagnostic et le traitement des intoxications radioactives (Vienne, 1962), de nombreuses synthèses relatives aux transuraniens ont été publiées [1-2]. Dans cette nouvelle revue, nous nous limiterons au devenir biologique des transuraniens inhalés, objet d'une attention particulière depuis l'essor de l'industrie nucléaire.

Nous avons préféré au mot métabolisme, l'expression devenir biologique, les transuraniens n'étant pas, au sens propre du terme, des métabolites mais de réels poisons.

Nous n'aborderons pas ce sujet de manière exhaustive, mais nous tenterons plutôt de nous focaliser sur certains points qui nous paraissent importants. L'exposé sera divisé en cinq chapitres dont l'ordre nous a paru proche de la chronologie habituelle observée lors d'une inhalation accidentelle de radioéléments. Le dépôt, le taux de rétention, puis la cinétique natureile de l'élimination et de la solubilisation des transuraniens seront d'abord abordés. Nous jetterons ensuite un bref regard sur quelques tentatives effectuées pour modifier le devenir biologique des transuraniens. Enfin, il nous paraît normal de terminer cette revue par un examen des problèmes nouveaux soulevés par les oxydes mixtes et les très fines particules.

\section{DÉPÔT}

Le dépôt de particules radioactives dans le tractus respiratoire a été décrit dans le rapport du Task Group on Lung Dynamics (TGLM) du Comité 2 de la C.I.P.R. (3). Il dépend, pour des particules grossièrement sphériques, uniquement de la taille aérodynamique des particules inhalées et non de la nature physicochimique de celles-ci. La majeure partie des études expérimentales effectuées avec les éléments transuraniens sont faites avec des particules de diamètre aérodynamique voisin du micromètre. Ceci favorise un dépôt profond alvéolaire, sans pour autant exclure un dépôt bronchique. Plus récemment, O. RAABE a précisé ce modèle pour le rat et le hamster [4]. Ces nouvelles données, quelque peu différentes, permettent pour les animaux de laboratoire les plus couramment employés de mieux affiner les estimations. 


\section{RÉTENTION}

Dans sa publication $n^{\circ} 19$, la C.I.P.R. décrit la rétention comme uniquement dépendante de la nature chimique du composé inhalé [5]. En prenant principalement pour exemple le plutonium, nous allons décrire quelques facteurs chimiques majeurs conditionnant le niveau de rétention, la cinétique d'épuration pulmonaire et le devenir biologique des transuraniens sous leur état de valence le plus stable.

\section{HYDROLYSE}

Le plutonium tétravalent est habituellement décrit comme un état de valence particulièrement hydrolysable conduisant, à $\mathrm{pH}$ voisin de 1,5 , à la formation rapide de colloïdes de poids moléculaire $10^{6}$ particulièrement insolubles [6]. Les constantes d'hydrolyse ou de formation de collö̈des sont

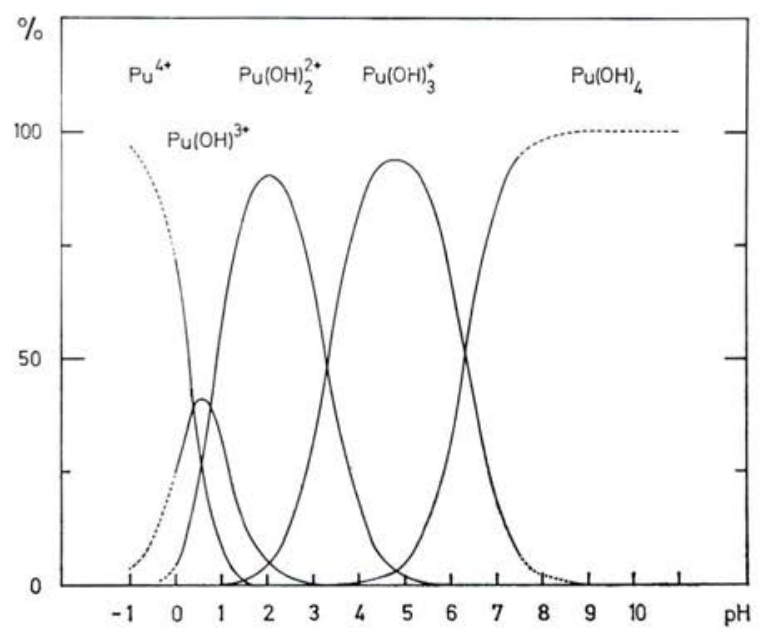

FIG. 1. - Pourcentages et domaines d'existence des différentes espèces du plutonium tétravalent en milieu non complexant de force ionique $1 . \log \mathrm{K}_{1}=-0,45 ; \log \mathrm{K}_{2}^{\dagger}=-0,75$; $\log \mathrm{K}_{3}=-3,3 ; \log \mathrm{K}_{4}=-6,3 ; \mathrm{C} 238_{\mathrm{Pu}}=10^{-7}-10^{-8} \mathrm{M}[9]$.

obtenues à des concentrations en plutonium toujours supérieures à $10^{-3}-10^{-4} \mathrm{M}$. A ces mêmes concentrations et à des acidités comprises entre 2 et $0,1 \mathrm{~N}, 10$ à 90 p. cent du plutonium tétravalent se dismute rapidement en $\mathrm{Pu}$ III et $\mathrm{Pu}$ VI [7]. Ces réactions sont particulièrement importantes dans la chimie du plutonium, mais la concentration envisagée est largement supérieure à celles que l'on peut généralement observer dans un organisme après une contamination accidentelle. En abaissant la concentration du plutonium à $10^{-7}-10^{-8} \mathrm{M}$, il est possible de ralentir considérablement la vitesse des réactions de dismutation et de polymérisation [7]. A ces concentrations, la méthode d'extraction

vol. $14-\mathrm{N}^{\circ}$ ! 
par solvant est la seule capable d'étudier l'hydrolyse et la complexation du plutonium tétravalent [8]. Les constantes d'hydrolyse obtenues par cette technique montrent que le plutonium tétravalent n'est pas totalement hydrolysé et demeure monomérique à $\mathrm{pH} 7$ [9] ( fig. 1).

Les premières constantes d'hydrolyse des transplutoniens trivalents déterminées par la même méthode (tableau I) montrent que le comportement biologique des ions $\mathrm{Am}^{3+}$, $\mathrm{Cm}^{3+}, \mathrm{Bk}^{3+}, \mathrm{Cf}^{3+}$ et $\mathrm{Es}^{3+}$ ne devrait pas être très différent. Par contre, la constante d'hydrolyse du $\mathrm{Fm}^{3+}$ laisse supposer que la transportabilité du $\mathrm{Fm}^{3+}$ sera intermédiaire entre les transplutoniens précédents et le plutonium tétravalent. Contrairement aux autres transuraniens, le neptunium forme à $\mathrm{pH} 7$ un ion neptunyl $\mathrm{NpO}_{2}{ }^{+}$relativement stable puisqu'il a été mis en évidence en milieu biologique [13].

\section{TABLEAU I}

Premières CONSTANTES D'HYDROLYSE, $\log \mathrm{K}_{1}$, DES TRANSPLUTONIENS TRIVALENTS (d'après Desire et al. [10] et HussonNoIs et al. [11-12])

\begin{tabular}{|c|c|c|c|c|c|}
\hline $\mathrm{Am}$ & $\mathrm{Cm}$ & $\mathrm{Bk}$ & $\mathrm{Cf}$ & $\mathrm{Es}$ & $\mathrm{Fm}$ \\
\cline { 1 - 3 }$-5,30$ & $-5,40$ & $-5,10$ & $-5,05$ & $-5,14$ & $-3,8$ \\
\hline
\end{tabular}

\section{FORMATION DE COMPLEXES}

En utilisant la technique d'extraction par solvant, il a été possible de décrire l'existence de dix complexes citriques différents de Pu IV (tableau II). Tous n'ont pas la même importance; trois complexes sont largement prépondérants $\mathrm{C}_{1}^{+}, \mathrm{C}_{1}^{-}, \mathrm{C}_{2}^{4-}$ selon la terminologie adoptée. Au niveau sanguin, c'est la forme $\mathrm{C}_{2}^{4-}$ correspondant à l'ion $\mathrm{Pu} \mathrm{O}(\mathrm{Cit})_{2}^{4-}$ qui sera véhiculée ( fig.2).

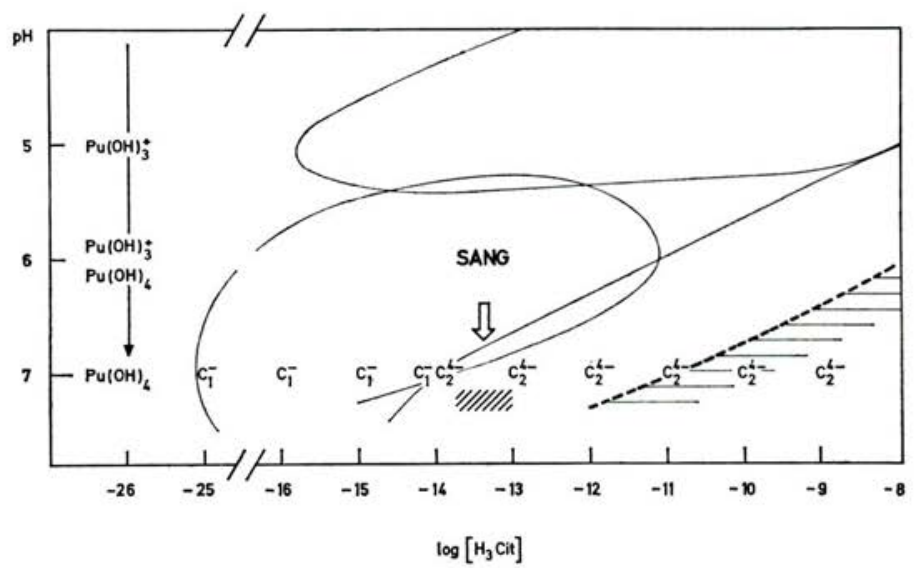

Fig. 2. - Domaine d'existence des principaux complexes de Pu IV en milieu citrique $[7,14]$. 
TABLEAU II

CONSTANTES DE FORMATION DES COMPLEXES CITRIQUeS DU PLUTONIUM TÉTRAVALENT [14]

$$
\begin{gathered}
\mathrm{C}_{l}^{z+} \equiv \mathrm{Pu}\left(\mathrm{H}_{3} \mathrm{Cit}\right)_{l} \mathrm{H}_{-y}^{(4-y)+}, \\
\mathrm{C}_{0}^{4+}+l \mathrm{H}_{3} \mathrm{Cit} \stackrel{\mathrm{K} l, y}{\rightleftarrows} \mathrm{C}_{l}^{(4-y)+}+y \mathrm{H}^{+} .
\end{gathered}
$$

\begin{tabular}{|c|c|c|c|c|}
\hline$l$ & $y$ & $\log \mathrm{K}_{l, y}$ & Charge & Symbole \\
\hline 1 & $\begin{array}{l}1 \\
2 \\
3 \\
4 \\
5\end{array}$ & $\begin{array}{l}+2,85 \\
+1,85 \\
+0,1 \\
-5,63 \\
-9,85\end{array}$ & $\begin{array}{l}3^{+} \\
2^{+} \\
1^{+} \\
0 \\
1^{-}\end{array}$ & $\begin{array}{l}\mathrm{C}_{1}^{3+} \\
\mathrm{C}_{1}^{2+} \\
\mathrm{C}_{1}^{+} \\
\mathrm{C}_{1}^{0} \\
\mathrm{C}_{1}^{-}\end{array}$ \\
\hline 2 & $\begin{array}{l}1 \\
2 \\
4 \\
6 \\
8\end{array}$ & $\begin{array}{l}+4 \\
+\quad 3,5 \\
+\quad 1,5 \\
-7,5 \\
-16,6\end{array}$ & $\begin{array}{l}3^{+} \\
2^{+} \\
0 \\
2^{-} \\
4^{-}\end{array}$ & $\begin{array}{l}\mathrm{C}_{2}^{3+} \\
\mathrm{C}_{2}^{2+} \\
\mathrm{C}_{0}^{2+} \\
\mathrm{C}_{2}^{2-} \\
\mathrm{C}_{2}^{4-}\end{array}$ \\
\hline
\end{tabular}

Le plutonium tétravalent est également complexé par l'acide isocitrique $10^{-6} \mathrm{M}$ [15]; par contıe, il précipite en présence d'acide cis aconitique et d'acide $\alpha$-cétoglutarique [7].

Les valeurs des constantes de formation de complexes citriques avec les transplutoniens montrent une grande similarité (tableau III) mais à $\mathrm{pH}$ voisin de 7. Les complexes citriques de $\mathrm{Cm}$ sont hydrolysés [17]. La similarité des différentes constantes laisse présager un comportement voisin pour les autres transplutoniens $\mathrm{M}^{3+}$.

TABLEAU III

VALEURS DES CONSTANTES DE FORMATION DES COMPLEXES CITRIQUES

\begin{tabular}{|c|c|c|c|c|c|c|}
\hline Complexe & $\mathrm{Am}$ & $\mathrm{Cm}$ & Bk & $\mathrm{Cf}$ & Es & $\mathrm{Fm}$ \\
\hline MH Cit $\mathrm{Cit}^{2}-\ldots$ & 10,76 & 10,69 & - & 10,90 & 10,64 & 11,17 \\
\hline $\mathrm{M} \mathrm{Cit}{ }_{2}^{3-} \ldots \ldots \ldots$ & 12,16 & 11,93 & - & 12,26 & 12,07 & 12,40 \\
\hline
\end{tabular}
DES ÉlÉments trivalents Am À Fm (d'après Hubert [16])

DISSOLUTION DES OXYDES

On dispose de peu de données relatives à la solubilisation des oxydes en milieu biologique. L'oxyde de plutonium 239 est classiquement considéré comme insoluble, toutefois, de nombreuses observations montrent qu'une lente dissolution des particules s'effectue au niveau pulmonaire. En utilisant le sérum synthétique sans fraction protéinique décrit par KaNAPILLY [18], 
Boyd et al. ont déterminé le taux de dissolution de ${ }^{239} \mathrm{PuO}_{2},{ }^{238} \mathrm{PuO}_{2}$ et ${ }^{241} \mathrm{AmO}_{2}$, tous trois obtenus à $1050^{\circ} \mathrm{C}$ et de diamètre aérodynamique voisin de $1 \mu \mathrm{m}$ [19]. Le plus soluble est ${ }^{238} \mathrm{PuO}_{2}: 1,2 \cdot 10^{-8} \mathrm{~g} \cdot \mathrm{cm}^{2} \cdot \mathrm{j}^{-1}$, puis ${ }^{241} \mathrm{AmO}_{2}: 4 \cdot 10^{-9}$, et ${ }^{239} \mathrm{PuO}_{2}: 6,5 \cdot 10^{-11}$. Ces résultats montrent bien que la forme chimique n'intervient pas seule dans les mécanismes de dissolution, mais qu'il existe aussi un facteur radioactif qui peut seul expliquer les différences de solubilisation des oxydes de plutonium 239 et 238. La solubilisation de ${ }^{238} \mathrm{PuO}_{2}$ s'effectue par arrachement de groupes d'atomes, environ vingt, lors du recul des noyaux résultant de la désintégration $\alpha$ [20-21]. Ce type de dissolution a été récemment confirmé in vivo par STRADLING [22].

\section{3. ÉPURATION PULMONAIRE}

Le modèle de la C.I.P.R. admet que l'épuration pulmonaire des transuraniens déposés dans les voies aériennes est très rapide et ne dépasse pas 1 à 2 jours quelle que soit la nature du composé inhalé. A l'opposé, le plutonium et autres transuraniens déposés dans la région alvéolaire sont retenus beaucoup plus longtemps et peuvent être classés en trois groupes, oxydes de plutonium, composés solubles de plutonium, autres transuraniens.

\section{OXYDES DE PLUTONIUM}

Le temps de demi-épuration varie pour l'oxyde de plutonium 239 de 150 à 500 jours chez le rat, de 300 à 1250 jours chez le chien [2]. L'épuration pulmonaire de ${ }^{238} \mathrm{PuO}_{2}$ est plus rapide, probablement à cause de l'instabilité de l'oxyde [23].

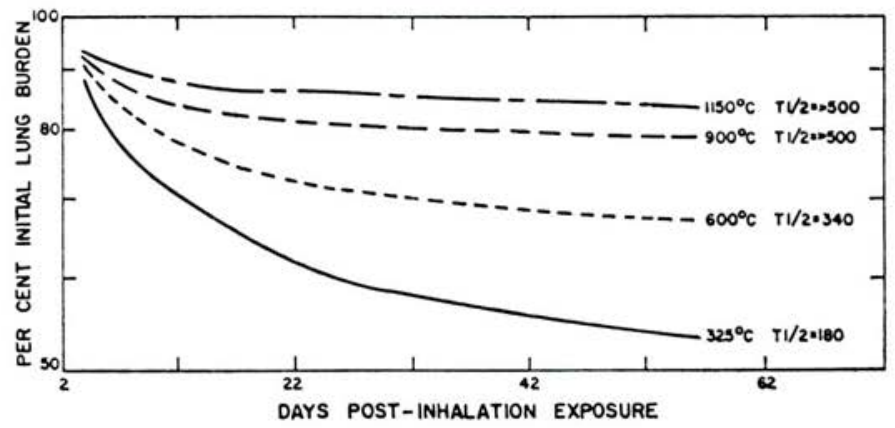

FIG. 3. - Épuration pulmonaire chez le chien après inhalation d'oxydes de plutonium 239 obtenus par calcination du chlorure à différentes températures (d'après J. MEWHINNEY [24]).

Certains facteurs physicochimiques, telle la température de calcination affectent l'épuration pulmonaire de l'oxyde de plutonium 239. MEWHINNEY [24] montre ainsi que le temps de demi-épuration $\left(\mathrm{T}_{1 / 2}\right)$ varie chez le chien de 180 à 500 jours lorsque la température de calcination de l'oxyde inhalé varie de 325 à $1150^{\circ} \mathrm{C}$ (fig. 3). 
Les oxydes obtenus à différentes températures n'ont pas la même surface spécifique. Les oxydes obtenus à basse température demeurent sous-stœchiométriques et renferment encore dans les réseaux cristallins des impuretés qui facilitent la dissolution de l'oxyde [25].

\section{TABLEAU IV}

VARIATION DU TEMPS DE DEMI-ÉPURATION ( $\left.\mathrm{T}_{1 / 2}\right)$ AVEC LE TEMPS D'OBSERVATION APRĖS INHALATION DE ${ }^{239} \mathrm{PuO}_{2}$ PAR LE SINGE BABOUIN [27]

\begin{tabular}{|l|c|c|c|}
\hline & $\begin{array}{c}\text { Temps } \\
\text { de survie } \\
\text { (jours) }\end{array}$ & $\begin{array}{c}\mathrm{T}_{1 / 2} \\
\text { (jours) }\end{array}$ & $\sigma$ \\
\cline { 1 - 3 } Groupe I.... & $<100$ & 134 & 63 \\
Groupe II... & $100-200$ & 476 & 188 \\
Groupe III ... & $<200$ & 755 & 584 \\
\hline
\end{tabular}

\section{TABLEAU $\mathrm{V}$}

Comparaison de la CHARge PUlMONAIRE EN PLUTONIUM APRĖS INHALATION DE DIFFÉRENTES FORMES CHIMIQUES

(résultats compilés par BAIR [2], d'après les travaux de LYUBCHANSKII [36])

\begin{tabular}{|c|c|c|c|c|}
\hline & & \multicolumn{3}{|c|}{$\begin{array}{l}\text { Charge pulmonaire } \\
\text { en p. cent de la charge initiale }\end{array}$} \\
\hline & & $\mathrm{pH}$ & 1 jour & 256 jours \\
\hline Citrate de plutonium ( $2 \%$ de citrate) & IV & 6,5 & 47 & 4 \\
\hline Triacétate de plutonyle......... & VI & 6,5 & 70 & 4 \\
\hline $\begin{array}{l}\text { Pentacarbonate de plutonium et } \\
\text { d'ammonium } \ldots \ldots \ldots \ldots \ldots \ldots\end{array}$ & IV & 7,4 & 85 & 6 \\
\hline Chlorure de plutonium ........... & III & 2 & 88 & 7 \\
\hline Nitrate de plutonium......... & IV & 2 & 74 & 9 \\
\hline
\end{tabular}

De nombreuses valeurs du temps de demi-épuration sont établies à partir de mesures effectuées en 60 jours ou moins [24, 26]. Si le temps d'observation est prolongé et porté à 200 jours, il a été montré avec des particules non toxiques de latex marqué au ${ }^{51} \mathrm{Cr}$, que pour le même animal (singe babouin) la valeur calculée de $T_{1 / 2}$ pouvait atteindre des valeurs supérieures à 1000 jours [27]. Cette augmentation de $T_{1 / 2}$ est également observée chez le babouin après inhalation de ${ }^{239} \mathrm{PuO}_{2}$ et n'est donc pas dépendante de la toxicité des particules [27] (tableau IV). Elle a été également observée chez le chien [28]. Si l'on compare ces résultats à ceux obtenus après contamination accidentelle chez l'homme [29], on remarque un commun allongement de la valeur de $T_{1 / 2}$ avec le temps. Ceci indique que l'épuration pulmonaire lente n'obéit pas à une fonction exponentielle simple mais plutôt à une fonction de puissance.

VOL. $14-\mathrm{N}^{\circ} 1$ 
Une récente comparaison des valeurs obtenues chez le chien et le singe [30] montre que la croissance de $T_{1 / 2}$ tend asymptotiquement vers une valeur limite voisine de 1000 jours. Cette valeur pourrait remplacer la valeur de 500 jours du modèle C.I.P.R.

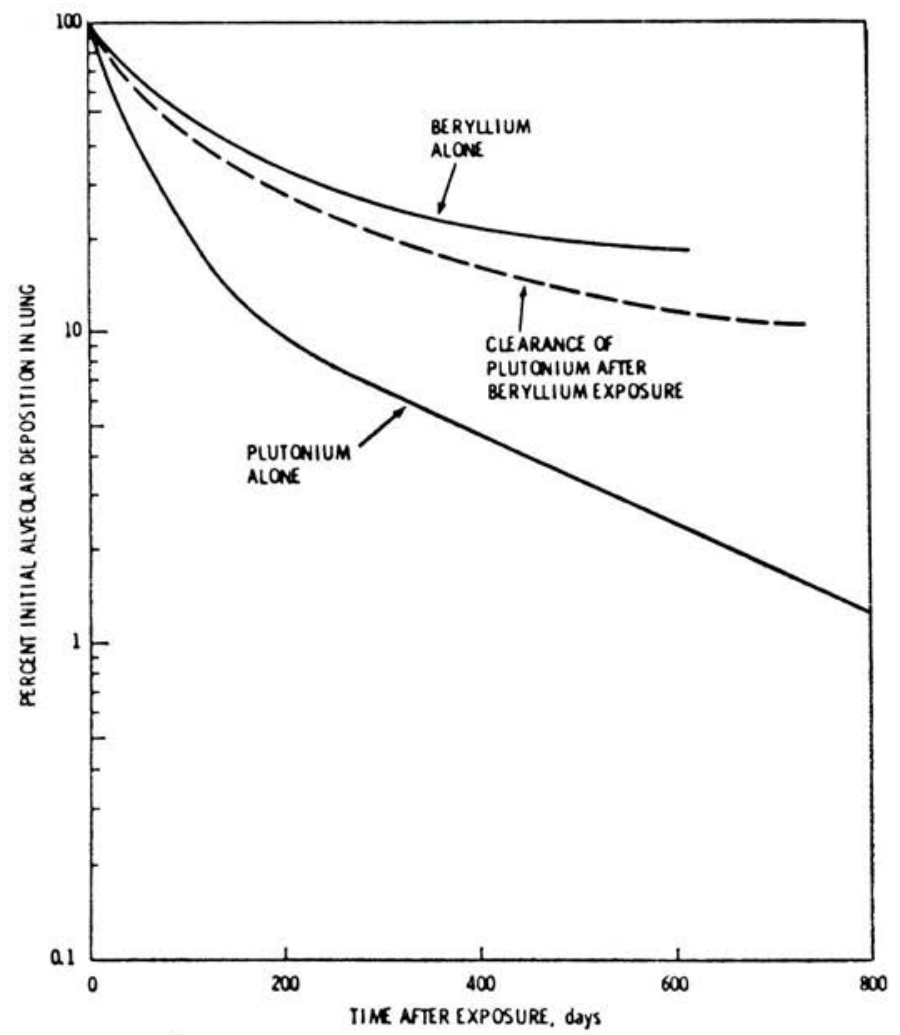

FIG. 4. - Épuration pulmonaire chez le rat d'oxyde de plutonium après inhalation d'oxyde de béryllium (SANDERS [34]).

L'épuration pulmonaire de l'oxyde de plutonium peut également être dépendante de la dose inhalée. Chez les rongeurs, NenOT puis SANDERS ont décrit un ralentissement de l'épuration pulmonaire lorsque la dose inhalée augmente $[31,32]$. Ceci correspond à la suppression de la phase intermédiaire $\mathrm{T}_{1 / 2} \simeq 30$ jours de l'épuration pulmonaire [33]. Le niveau de dose supprimant cette phase intermédiaire semble varier chez le rat d'une lignée à l'autre.

Enfin, l'épuration pulmonaire de l'oxyde de plutonium 239 est considérablement ralentie lorsque l'inhalation de l'oxyde suit une inhalation d'oxyde de béryllium [34] ( fig. 4), ce ralentissement est à associer aux modifications de populations cellulaires intraalvéolaires produites par l'inhalation de béryllium [35]; il pose, au niveau de l'épuration pulmonaire, le problème des associations de toxiques respiratoires et doit être pris en considération lors des extrapolations à l'homme. 


\section{Composés SOLUBLES DU PLUTONIUM}

Les composés solubles du plutonium sont épurés du poumon plus rapidement que les oxydes. Le mécanisme d'épuration est double; par voie macrophagique classique mais aussi et principalement par passage de la barrière alvéolocapillaire. La forme chimique qui conditionne les mécanismes d'hydrolyse et de complexation joue un rôle important dans la cinétique d'épuration pulmonaire. Toutefois, comme le montre le tableau $\mathrm{V}$, les niveaux pulmonaires résiduels restent très voisins 256 jours après l'inhalation, quelle que soit la forme chimique soluble du plutonium inhalé [36].

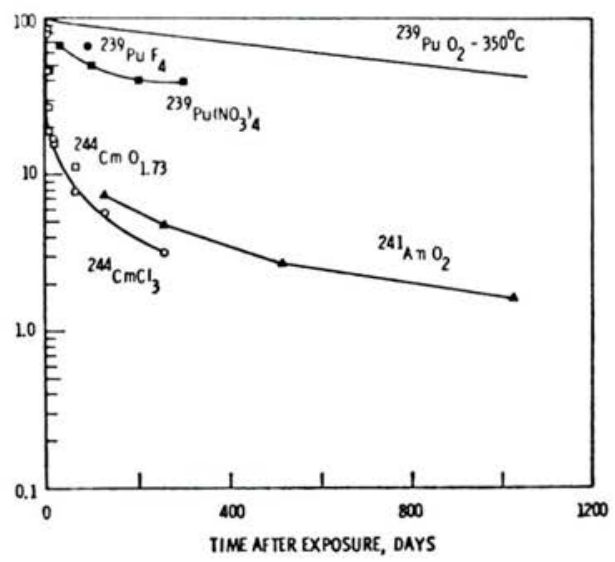

FIG. 5. - Épuration pulmonaire chez le chien de composés d'américium et de curium exprimée en p. cent de la charge initiale (d'après MCCLeLlan [38]).

Après inhalation par le rat d'environ $5 \mu \mathrm{Ci}$ de nitrate de plutonium à $\mathrm{pH} 1,5$, NENOT et al. ne constatent pas de différence majeure entre les isotopes 238 et 239 au niveau pulmonaire [37]. Ce résultat diffère de ceux obtenus par les mêmes auteurs après injection intramusculaire (élimination beaucoup plus rapide de l'isotope 238). Ceci ne peut s'expliquer que par une différence du taux de complexation lié à la masse injectée, variant d'un facteur 100 à 200 pour la même activité des deux isotopes, au niveau des sites d'entrée. Le pouvoir complexant du poumon se révèle ici moins élevé que celui du muscle.

\section{AUtres TRANSURANIENS}

Les oxydes d'américium, de curium et d'einsteinium sont éliminés du poumon beaucoup plus rapidement que les oxydes de plutonium 238 et 239 car, probablement, ils sont plus solubles [38-39]. Leur solubilité est telle qu'il n'est pas noté chez le chien de différence sensible de l'épuration pulmonaire entre l'oxyde et le chlorure de curium 244 [38] ( fig. 5).

Toutefois, l'épuration pulmonaire rapide de ces éléments s'accompagne d'une épuration très lente d'un compartiment résiduel comme le montre la 
figure 6. Ce phénomène a été observé chez le rat pour tous les transuraniens; l'importance de ce résidu varie de 1 à $4 \mathrm{p}$. cent de la charge initiale et s'élimine avec une valeur de $T_{1 / 2}$ variant de 250 à 500 jours (tableau VI).

TABLEAU VI

NIVEAU ET PÉRIODE D'ÉPURATION DU COMPARTIMENT RÉSIDUEL CHEZ LE RAT APRÈS INHALATION DE TRANSURANIENS (d'aprèS LAFUMA [41])

\begin{tabular}{|c|c|c|c|}
\hline & \multirow{2}{*}{$\underset{\substack{\text { principal } \\
\text { du }}}{\mathrm{T}_{1 / 2}}$} & \multicolumn{2}{|c|}{ Compartiment résiduel } \\
\hline & & $\begin{array}{l}\text { P. cent } \\
\text { de la charge } \\
\text { initiale }\end{array}$ & $\mathrm{T}_{1 / 2}$ \\
\hline${ }^{238} \mathrm{PuO}_{2} \ldots \ldots \ldots \ldots \ldots \ldots$ & 30 & 4 & 500 \\
\hline${ }^{238} \mathrm{Pu}\left(\mathrm{No}_{3}\right)_{4} \ldots \ldots \ldots \ldots \ldots \ldots$ & 15 & 2 & 250 \\
\hline${ }^{239} \mathrm{PuO}_{2} \ldots \ldots \ldots \ldots \ldots \ldots$ & 120 & 4 & 500 \\
\hline${ }^{239} \mathrm{Pu}\left(\mathrm{NO}_{3}\right)_{4} \ldots \ldots \ldots \ldots \ldots$ & 60 & 2 & 250 \\
\hline${ }^{241} \mathrm{AmO}_{2} \ldots \ldots \ldots \ldots \ldots \ldots$ & 12,5 & 1 & 250 \\
\hline${ }^{241} \mathrm{Am}\left(\mathrm{NO}_{3}\right)_{3} \ldots \ldots \ldots \ldots \ldots$ & 12,5 & 1,5 & 250 \\
\hline${ }^{244} \mathrm{Cm}\left(\mathrm{NO}_{3}\right)_{3} \ldots \ldots \ldots \ldots \ldots$ & 8 & 1 & 250 \\
\hline
\end{tabular}

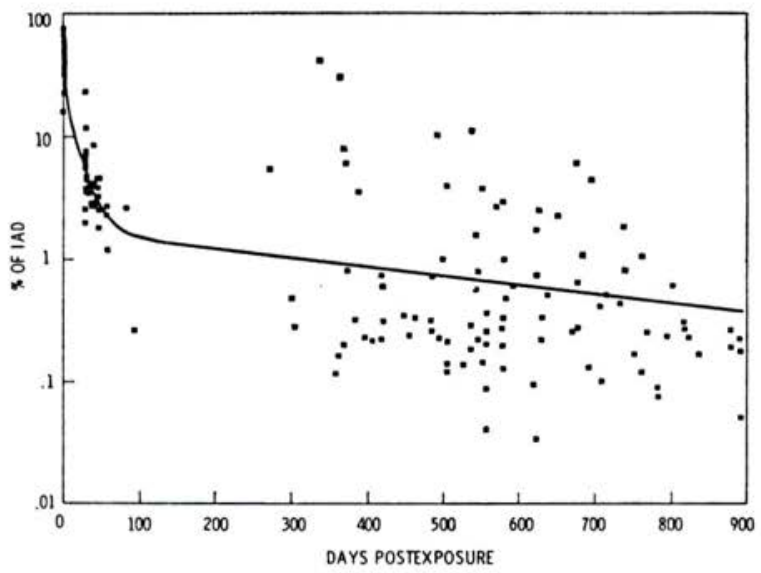

Fig. 6. - Mise en évidence chez le rat d'un compartiment résiduel à épuration lente après inhalation d'oxyde de curium 244 (d'après MAHAFFEY [40]).

Ce résidu correspond à une localisation particulière des radioéléments. Ainsi, au niveau de l'épithélium bronchique, une concentration de traces $\alpha$ a été mise en évidence par autoradiographie 600 jours après inhalation de nitrate de curium [42]. La dose délivrée au poumon par ce résidu est négligeable, mais doit être prise en considération si les effets doivent être rapportés aux doses délivrées aux cellules sensibles. 


\section{TRANSLOCATION}

Le mot anglais translocation étant couramment employé et plus évocateur que les équivalents français migration ou transportabilité, nous le conserverons dans cette revue.

Le sang circulant distribue les transuraniens après leur solubilisation au niveau pulmonaire dans les différents tissus de l'organisme. La distribuiion relative de ces composés au niveau des organes dépend essentiellement de la forme chimique inhalée mais surtout de la stabilité des complexes présents au niveau sanguin. Le complexe avec la transferrine est la forme circulante prédominante pour le plutonium tétravalent [43]. Inversement, sur la base d'études par filtration sur gel de Sephadex, Popplewell et Boocock [44] ont montré que l'américium est peu complexé par la transferrine humaine et que le curium. III ne l'est pas. Ces deux éléments et probablement les autres transuraniens forment des complexes faibles avec d'autres protéines sériques en compétition avec les complexes citriques, elimines par le rein [45]. L'équilibre entre ces deux formes circulantes peut être déplacé en faveur des complexes citriques par perfusion d'ions citrate. Sur la base d'observations bibliographiques, MASSE [46] a pu établir que les complexes les moins stables formés avec les protéines se fixent dans le foie après leur dissociation, les complexes plus forts qui ont résisté au filtre hépatique se déposant au niveau osseux. D'autre part, les complexes citriques se fixent préférentiellement dans le squelette, compte tenu de la différence des débits sanguins.

Une étude autoradiographique a mis en évidence la dissolution alvéolaire d'oxyde d'américium au niveau des vacuoles de pinocytose, la forme soluble diffusible pourra migrer vers le sang puis s'associer éventuellement avez des protéines sériques [47].

La translocation dépendant de la solubilisation des éléments inhalés et de la forme chimique véhiculée, nous allons retrouver dans ce chapitre la classification décrite précédemment : oxydes insolubles, composés solubles du plutonium, autres transuraniens. Les données bibliographiques étant très nombreuses, nous ne décrirons ici que quelques exemples représentatifs des trois classes.

Après inhalation par des chiens d'oxyde de plutonium 239 insoluble, la charge des ganglions thoraciques, du foie et du squelette atteint après 5 ans un plateau situé aux environs de $30-40$ p. cent, 15 p. cent, 4 à 5 p. cent respectivement de la dose inhalée [48]. Trois ans après inhalation d'oxyde de plutonium 239, la charge hépatique du babouin est inférieure à celle du chien [49]. Cette différence observée également chez les rongeurs après inhalation de citrate de cérium 144 [50] peut être le résultant d'une répartition hépatique en deux compartiments ne s'épurant pas à la même vitesse. Le taux de rétention de ces deux compartiments varierait selon DURBIN [51] d'une espèce à l'autre.

Les vitesses de diffusion du plutonium dépendent de la solubilité de l'oxyde. La migration est plus rapide pour les oxydes obtenus à basse tempé-

VOL. $14-\mathrm{N}^{\circ} 1$ 
rature (tableau VII). Pour un même oxyde, la charge hépatique augmente avec la dose inhalée [52]. La translocation est beaucoup plus importante après inhalation d'oxyde de plutonium 238, l'os devenant dans ce cas l'organe critique [53]. La migration des autres transuraniens est encore plus rapide.

\section{TABLEAU VII}

Distribution EN P. CENT DE LA CHARge initiale DU PLUTONIUM 239 CHEZ LE CHIEN 56 JOURS APRÈs L'INHALATION D'OXYDES (d'après MEWHiNNEY [23])

\begin{tabular}{|c|c|c|c|c|}
\hline $\begin{array}{c}\text { Température } \\
\text { d'obtention } \\
\text { de l'oxyde } \\
\left({ }^{\circ} \mathrm{C}\right)\end{array}$ & Poumon & $\begin{array}{c}\text { Ganglions } \\
\text { thoraciques }\end{array}$ & Foie & Squelette \\
\hline $325 \ldots \ldots \ldots \ldots$ & 54 & 0,4 & 5,9 & 7,6 \\
$600 \ldots \ldots \ldots \ldots$ & 67 & 0,6 & 1,3 & 2,1 \\
$900 \ldots \ldots \ldots \ldots$ & 80 & 0,4 & - & 0,2 \\
$1150 \ldots \ldots \ldots \ldots$ & 87 & 0,5 & - & 0,2 \\
\hline
\end{tabular}

TABLEAU VIII

Translocation de L'OXyde d'Américium INHALÉ CHEZ LE CHIEN, EXPRIMÉE EN P. CENT DE LA CHARGE CORPORELLE

(tableau établi par BAIR [2])

\begin{tabular}{|c|c|c|c|c|c|c|c|}
\hline Jours ...... & $127(*)$ & $256(*)$ & $512(*)$ & $1022(*)$ & $30(* *)$ & $90(* *)$ & $270(* *)$ \\
\hline Poumon.......... & 9,9 & 6,8 & 3,6 & 2,6 & $55-53$ & $29-44$ & $14-17$ \\
\hline Foie.......... & 41 & 51 & 34 & 24 & $19-23$ & $39-21$ & $32-49$ \\
\hline Os............. & 47 & 39 & 59 & 70 & $11-17$ & $18-24$ & $45-31$ \\
\hline $\begin{array}{c}\text { Ganglions bron- } \\
\text { chiques........ }\end{array}$ & 0,25 & 0,14 & 0,13 & 0,0005 & - & - & - \\
\hline Charge corporelle & 24 & 15 & 20 & 15 & $0,11-1,3$ & $0,12-1,2$ & $0,15-0,59$ \\
\hline
\end{tabular}

(*) D’après Thomas et al. [39]. (**) D'après Craig et al. [54].

Le tableau VIII montre les valeurs obtenues chez le chien après inhalation d'oxyde d'américium. La comparaison des deux expériences montre que jusqu'à 270 jours les charges hépatique et osseuse sont semblables mais qu'audelà de 500 jours apparaît une prépondérance de la charge osseuse. L'os est également l'organe critique pour les autres transplutoniens. 


\section{ESSAIS DE MODIFICATION DU DEVENIR BIOLOGIQUE}

Différentes thérapeutiques ont été mises au point pour diminuer la charge corporelle après inhalation des transuraniens: la chélation par le DTPA pour les éléments solubles, le lavage pulmonaire pour les insolubles. En marge de ces techniques discutées lors du dernier congrès de Vienne en 1975, nous soulignerons ici deux points importants observés après inhalation d'oxyde de plutonium 239.

L'élimination naturelle du plutonium se faisant les premiers jours principalement par remontée macrophagique, certains auteurs ont émis l'hypothèse que l'efficacité de cette épuration peut être améliorée en augmentant le nombre des macrophages. Des essais par trois techniques différentes - injection intraveineuse de l'adjuvant complet de Freund, intratrachéale de Zymosan, intramusculaire d'un lysat de bactéries - ont malheureusement infirmé cette hypothèse et apportent même un effet aggravant puisque le taux de rétention à la fin du traitement (100 jours) représente respectivement 158,132 et 107 p. cent de la charge pulmonaire des animaux témoins [55]. Parallèlement, l'emploi de modificateurs de sécrétions bronchiques aussi divers que $\mathrm{NaCl}$, $\mathrm{AgNO}_{3}$, le chlore gazeux, les ions négatifs, le glycol ou le bissolvon, n'a jamais modifié favorablement les cinétiques d'épuration lente de l'oxyde de plutonium [55-56]. L'emploi de telles méthodes chez l'homme [57] semble donc à proscrire puisqu'apportant parfois, chez l'animal, un effet aggravant.

Il est possible, chez le babouin, d'éliminer par lavage pulmonaire une fraction pratiquement constante d'oxyde de plutonium 239 inhalé, du dixième jusqu'au centième jour suivant l'inhalation [58]. Inversement après lavages répétés selon la technique décrite par NoLiBE [59], l'accessibilité du plutonium diminue considérablement, mais peut être accompagnée d'une accélération de l'épuration pulmonaire se poursuivant après l'arrêt du traitement [60]. Ceci correspond probablement à une migration interstitielle favorisée par la disjonction des cellules de la paroi alvéolaire durant les 24 heures suivant le lavage [61]. Cette modification de localisation peut être la cause d'un allongement de la durée de vie des animaux lavés supérieur à celui attendu par la seule diminution de dose obtenue grâce au lavage [62]. Ce résultat est à rapprocher des expériences décrites par RichmoND [63] montrant que la toxicité des particules d'oxyde de plutonium 239 peut dépendre de leur localisation, puisqu'elle diminue lorsque ces particules sont retenues au niveau des capillaires après injection intraveineuse.

\section{OXYDES MIXTES ET PARTICULES NANOMÉTRIQUES}

Les données décrites dans les chapitres précédents sont toutes dérivées d'études d'inhalation d'un seul composé, soluble ou insoluble, sous forme de particules ou goutelettes de diamètre voisin du micromètre. Depuis le nouvel essor de l'industrie nucléaire, l'accent est porté sur les oxydes mixtes $(\mathrm{U}, \mathrm{Pu}) \mathrm{O}_{2}$ utilisés dans les réacteurs, mais aussi sur les oxydes mixtes, tel $(\mathrm{Pu}, \mathrm{Na}), \mathrm{O}_{x}$ pouvant résulter d'un accident éventuel lié à l'exploitation des surrégénérateurs.

voL. $14-\mathrm{N}^{\circ} 1$ 
Dans le cycle des combustibles nucléaires, l'oxyde d'uranium est sous forme $\mathrm{UO}_{2}$. La chimie de l'uranium est cependant compliquée par la présence d'un grand nombre d'oxydes non-stœchiométriques qui affectent le réseau cristallin et la solubilité de l'oxyde. Lors d'un accident industriel, où les oxydes

\section{TABLEAU IX}

Charges hépatiques et osseuses en Plutonium, exprimées en P. CENT DE LA CHARGE PULMONAIRE INITIALE APRÈS INHALATION PAR DES SOURIS D'UN AÉROSOL MIXTE Na-Pu $(\mathrm{Na}: \mathrm{Pu}=1,3)$ (d'après BRIGHTWELL [65])

\begin{tabular}{|c|c|c|}
\hline Jours & Foie & Squelette \\
\cline { 2 - 3 } & \multirow{2}{*}{$\ldots \ldots \ldots \ldots$} & $0,071>->0,73$ \\
$2 \ldots \ldots \ldots \ldots$ & 1,46 & 2,22 \\
$6 \ldots \ldots \ldots \ldots$ & 1,41 & 2,49 \\
$14 \ldots \ldots \ldots \ldots$ & 0,90 & 3,69 \\
$35 \ldots \ldots \ldots \ldots$ & 0,51 & 7,8 \\
\hline
\end{tabular}

d'uranium et de plutonium sont en solution solide, la solubilité d' $\mathrm{UO}_{2}-\mathrm{PuO}_{2}$ sera celle de l'oxyde d'uranium. Il en résulte que l'on doit s'attendre à une augmentation de la solubilité, donc de la translocation de l'oxyde de plutonium lorsque celui-ci est issu d'une contamination mixte avec l'oxyde d'uranium [64].

Les risques nouveaux apportés par l'emploi du sodium dans les réfrigérants des réacteurs surrégénérateurs ont fait l'objet d'études toxicologiques d'abord effectuées simultanément par le National Radiclogical Protection Board (Harwell, U. K.) et l'Institut de Protection et de Sûreté nucléaire (S.P.T.E., Bruyères-le-Châtel). Les techniques employées par les deux laboratoires européens s'appuient sur des conceptions différentes, mais complémentaires, d'un éventuel accident. La technique anglaise - explosion par décharge électrique d'une spire de plutonium métallique enrobé de sodium - tend à recréer, malgré l'utilisation de plutonium métal et non d'oxyde, l'accident explosif libérant du combustible dans l'atmosphère. La technique française - étude des composés obtenus par la combustion, en présence d'oxyde de plutonium de sodium préalablement chauffé à $500^{\circ} \mathrm{C}$ - se rapproche plus d'un feu de sodium contaminé.

Les oxydes de plutonium obtenus par la technique d'explosion traversent rapidement la barrière pulmonaire pour se fixer à long terme au niveau osseux (tableau IX). Le taux de translocation augmente avec le rapport $\mathrm{Na}: \mathrm{Pu}$, pour atteindre un plateau lorsque le rapport $\mathrm{Na}: \mathrm{Pu}$ est d'environ 20 [65-66]. On remarque également que le plutonium issu d'un tel aérosol franchit cent fois plus la barrière pulmonaire qu'un oxyde habituel [67] et qu'une fraction importante peut être éliminée par voie urinaire [66], contrairement à ce qui a été décrit précédemment pour les oxydes de plutonium. La solubilité et la translocation de l'oxyde de plutonium préparé par cette technique ont pu être 
associées à l'identification de particules nanométriques rapidement solubles qui traversent aisément les barrières pulmonaires et rénales [68] (tableau X).

\section{TABLEAU $X$}

DEVENIR DU PLUTONIUM INHALÉ A L'ÉTAT PARTICULAIRE DE DIFFÉRENTES TAILLES OU SOUS FORME DE COMPLEXE CITRIQUE 17 A 21 JOURS APRÈS LA CONTAMINATION (d'après Smith [69] et Stradling [67])

\begin{tabular}{|c|c|c|c|c|}
\hline & $\begin{array}{c}\mathrm{PuO}_{2} \\
0,025-0,22 \mu \mathrm{m}\end{array}$ & $\begin{array}{c}\mathrm{PuO}_{2} \\
0,001 \mu \mathrm{m}\end{array}$ & $\begin{array}{c}\mathrm{Pu}: \mathrm{Na}, 1: 20 \\
0,001 \mu \mathrm{m}\end{array}$ & $\begin{array}{c}\text { Citrate } \\
\text { de } \\
\text { plutonium }\end{array}$ \\
\hline Poumon............ & 92 & 20 & 18 & 7,4 \\
\hline Foie $\ldots \ldots \ldots \ldots \ldots$ & N.D & 4,5 & 6,3 & 6,4 \\
\hline Carcasse........... & N.D & 48 & 47 & 67 \\
\hline Urine $\left({ }^{*}\right) \ldots \ldots \ldots$ & N.D & 9,3 & 11,3 & 3,6 \\
\hline Fèces $(*) \ldots \ldots \ldots$. & 6 & 15,4 & 16 & 13,7 \\
\hline
\end{tabular}

(*) Valeurs cumulées.

La présence de ces particules nanométriques n'est pas uniquement associée à la présence de sodium puisqu'elles sont mises en évidence lors de l'explosion d'un fil de plutonium par la même technique [69]. Ces particules nanométriques sont également la cause probable d'une élimination urinaire élevée après inhalation d'un mélange issu de la combustion d'un alliage $\mathrm{Pu}-\mathrm{Mg}$ dont l'ignition est déclenchée par un arc électrique [70]. STRADLing a montré par ailleurs que la dissolution et la translocation de l'oxyde de plutonium 238 étaient dues à la présence de ces mêmes particules résultant de l'arrachement d'une vingtaine d'atomes par recul des noyaux de désintégration $\alpha$ [22].

La migration du plutonium après contamination par des composés issus de la combustion du sodium en présence d'oxyde de plutonium ne peut être associée uniquement à la taille des particules présentes lors de tels incendies. Il a été, en effet, montré que la fraction solubilisée dans l'eau, du résidu de combustion, migrait vers l'os tout aussi rapidement sans être toutefois accompagnée d'une élimination urinaire [71]. Cette fraction a été identifiée in vivo comme étant une forme oxydée du plutonium [72].

\section{CONCLUSION}

De cette revue sur le devenir biologique des transuraniens nous n'extrairons que certains faits qui nous paraissent importants et les conclusions qui en découlent.

Il ressort de cette étude que la rétention, l'épuration pulmonaire et la translocation dépendent bien de la chimie des transuraniens inhalés comme le décrit la C.I.P.R. Toutefois, nous avons pu remarquer que la nature chimique n'était pas le seul paramètre puisque le devenir biologique des oxydes de

vol. $14-\mathrm{N}^{\circ} 1$ 
plutonium 239 et 238 diffère notablement. Les expériences de STRADLING ont démontré in vivo que la radioactivité spécifique de l'isotope 238 est responsable de la solubilisation de l'oxyde par formation de particules nanométriques. L'importance de ces particules nanométriques, rencontrées par ailleurs avec l'isotope 239, est considérable puisque leur devenir biologique semble, à ce jour, bouleverser tous les modèles métaboliques établis. L'exemple de l'oxyde de plutonium 239 est révélateur; la valeur du temps de demi-épuration recommandée par la C.I.P.R., 500 jours, qui pourrait être prolongée à 1000 jours si l'on ne tient compte que des particules microniques, ne correspond plus aux valeurs réelles pour les particules nanométriques. L'origine de ces particules reste à établir puisque si elles sont révélées par combustion simultanée de plutonium et de sodium, elles sont également présentes lors de la combustion du plutonium seul, ou de l'alliage plutonium-magnésium. L'importance relative de ces particules dans les différents cas de contamination atmosphérique est à préciser afin de définir un nouveau schéma d'épuration. La faculté de transfert particulièrement étonnante de ces particules pose un réel problème de risque tératogène car, franchissant facilement la barrière rénale, nous n'avons plus l'assurance que la barrière placentaire reste un filtre absolu.

Il ressort, dès maintenant, que l'oxyde de plutonium 239 ne peut être, en toutes occasions, assimilé à un composé insoluble. Son mélange avec l'oxyde d'uranium peut faciliter sa solubilisation et la présence de particules nanométriques bouleverse les modèles classiques. Il peut également réagir avec le sodium pour conduire, lors d'un accident, à la formation de composés de valence supérieure dont le devenir biologique est relativement mal connu.

Un deuxième point, l'épuration bronchique, mérite également un examen nouveau. Elle est considérée par la C.I.P.R. comme rapide. Certaines observations sur le curium décrites précédemment semble infirmer cette hypothèse. Sur la base d'études effectuées avec des éléments autres que les transuraniens, il semble que la valeur C.I.P.R. surestime l'épuration bronchique [73]. Elle ne tient pas compte du fait qu'une fraction variable des particules s'épure plus lentement [74-75], ni qu'une fraction des particules peut pénétrer directement l'épithélium alvéolaire [76]. Si l'on considère la dose délivrée au poumon, ce phénomène peut être négligé, mais si l'on ne considère uniquemeni que les cellules à risque, le modèle actuel sous-estime l'irradiation bronchique [77].

Il reste, cependant, que malgré certains points à mieux préciser, l'importance des expériences sur le devenir biologique des transuraniens permet, dans un nombre de cas important, de définir assez bien les modèles " métaboliques " à la base des évaluations de risques.

\section{REMERCIEMENTS}

L'auteur tient à remercier le Professeur V. Volf (Karlsruhe) et le Docteur H. Smith (Harwell) qui ont suscité cette revue, mais surtout le Docteur R. MASSE pour son apport original tant dans la conception de cet article que dans la discusision de points particuliers du métabolisme des transuraniens. 


\section{BIBLIOGRAPHIE}

[1] Hodge H. C., Stannard J. N., Hursh J. B. Uranium, plutonium, transplutonic elements, Handbook of Experimental pharmacology, 36. Berlin, Springer Verlag, 1973.

[2] BAIR W. J. Recent animal studies on the deposition, retention and translocation of plutonium and other transuranic compounds. In Diagnosis and Treatment of incorporated radionuclides, Vienne, 8-12 décembre 1975. Vienne, I.A.E.A., 1976, 51-83.

[3] I.C.R.P. Task Group on Lung Dynamics (Morrow P. E., Chairman), Deposition and retention models for internal dosimetry of the human respiratory tract. Health Phys., 1966, 12, 173-207.

[4] Raabe O. G., Yeh H. C., Newton G. J., Phalen R. F., Velasquez D. J. Deposition of inhaled monodisperse aerosols in small rodents. In : Inhaled Particles IV, (Walton W. H., Ed.). Oxford, Pergamon Press, 1977, 1, 3-21.

[5] I.C.R.P. Publication 19, The metabolism of compounds of plutonium and other actinides. Oxford, Pergamon Press, 1972, p. 6.

[6] Cleveland J. M. The Chemistry of Plutonium. New York, Gordon and Breach, 1970 , p. 81.

[7] Métrvier H. Contribution à l'étude de l'hydrolyse du plutonium tétravalent et de sa complexation par des acides d'intérêt biologique, thèse, Paris 1973. Rapport CEA-R-4477.

[8] Guillaumont R., Muxart R., Bouissières G. Extraction par solvant d'un élément à l'état de chélate. Bull. Soc. chim. Fr., 1968, 5, 1952-1956.

[9] Métivier H., Guillaumont R. Hydrolyse du plutonium tétravalent. Radiochem. Radioanal. Letters, 1972, 10, $\mathrm{n}^{\circ} 2$ 2, 27-35.

[10] Desire B., Hussonnois M., Guillaumont R. Détermination de la première constante d'hydrolyse de l'américium, du curium, du béryllium et du californium. C. R. Acad. Sc., Paris, 1969, 269, 448-451.

[11] Hussonnois M., Hubert S., Aubin L., Guillaumont R., Bouissières G. Détermination de la première constante d'hydrolyse du fermium. Radiochem. Radioanal. Letters, $1972,10, \mathrm{n}^{\circ} 4,231-238$.

[12] Hussonnois M., Hubert S., Brillard L., Guillaumont R. Détermination de la première constante d'hydrolyse de l'einsteinium. Radiochem. Radioanal. Letters, 1973, $15, \mathrm{n}^{\circ} 1,47-56$.

[13] Masse R., Jovanovic V., Fragu P., L'Hullier I., Lafuma J. Utilisation de l'électrophorèse en rideau pour la détermination de l'état physicochimique des radioéléments et des composés radioactifs en solution dans les liquides biologiques, fer, cérium, neptunium, calcitonine. Rapport CEA-R-4417, 1972.

[14] Métivier H., Guillaumont R. Complexes citriques du plutonium tétravalent. Radiochem. Radioanal. Letters, 1972, 10, $\mathrm{n}^{\circ} 4,239-250$.

[15] Métivier H., Guillaumont R. Complexes isocitriques du plutonium tétravalent. Radiochem. Radional. Letters, 1972, 11, $\mathrm{n}^{\text {os }} 3-4,165-169$.

[16] Hubert S., Hussonnois M., Brillard L., Goby G., Guillaumont R. Détermination simultanée de constantes de formation de complexes citriques de l'américium, du curium, du californium, de l'einsteinium et du fermium. J. Inorg. Nucl. Chem., 1974, 36, 2361-2366.

[17] Moutte A., Guillaumont R. Complexes citriques d'actinium et de curium. Rev. Chimie Min., 1969, 6, 603-610.

voL. $14-\mathrm{N}^{\circ} 1$ 
[18] Kanapilly G. M., Raabe O. G., Goh C. T. H., Chimenti R. A. Measurement of in vitro dissolution of aerosol particles for comparison to in vivo dissolution in the lower respiratory tract after inhalation. Health Phys., 1973, 24, 497-507.

[19] Boyd H. A., RaAbe O. G., Peterson P. K. Production of monodisperse respirable aeorols of ${ }^{241} \mathrm{AmO}_{2}$ and evaluation of in vitro dissolution. Inhalation Toxicology Research Institute, annual report 1973-1974, LF-49, 1974, 1-4.

[20] Fleischer R. L. On the "dissolution" of respirable $\mathrm{PuO}_{2}$ particles. Health Phys., 1975, 29, 69-73.

[21] Fleischer R. L., RaAbe O. G. Fragmentation of respirable $\mathrm{PuO}_{2}$ particles in water by alpha decay, a mode of dissolution. Health Phys., 1977, 32, 253-257.

[22] Stradling G. N., Ham G. J., Smith H., Cooper J., Breadmore S. E. Factors affecting the mobility of plutonium 238 dioxide in the rat. Int. J. Radiat. Biol., 1978, 34, 37-47.

[23] Ballou J. E., Craig D. K., Park J. F., Ragan H. A., Sanders C. L. Dose-effect studies with inhaled plutonium in rats and dogs. Pacific Northwest Laboratory annual report for 1972, BNWL-1750, 1, 1973, 21-27.

[24] Mewhinney J., Muggenburg B. A., McClellan R. O., Miglio J. J. The effect of varying physical and chemical characteristics of inhaled plutonium aerosols on meta, bolism and excretion. In : Diagnosis and treatment of incorporated radionuclides, Vienne, 8-12 décembre 1975. Vienne, I.A.E.A., 1976, 87-97.

[25] BoesCh A. Communication personnelle.

[26] Métivier H., Nenot J. C., Masse R., Nolibe D., Lafuma J. Étude clinique de l'épuration pulmonaire chez le singe et le chien. C. R. Acad. Sc., Paris, série D, 1974, 278, 671-674.

[27] Métivier H., Masse R., Nolibe D., Lafuma J. Effect of time on the determination of the clearance rates of insoluble plutonium 239 oxide. Health Phys., 1977, 32, 447-450.

[28] BAIR W. J. Toxicology of plutonium, In : Advances in radiation biology, 1974, 4, 255-315.

[29] WatTS L. Clearance rates of insolubles plutonium 239 compounds from the lung. Health Phys., 1974, 29, 53-59.

[30] Bair W. J., Métivier H., Park J. F., Masse R., Stevens D. L., Lafuma J., Watson C. R., NoLibe D. Comparison of acute mortality in baboons and dogs after inhalation of ${ }^{23}{ }^{9} \mathrm{PuO}_{2}$. Radiat. Res. (in press).

[31] NeNOT J. C. Étude de l'influence de l'irradiation sur l'épuration pulmonaire. In : Inhaled Particles III (Walton W. H., Ed.). Old Woking, Unwin Brothers Ltd, 19711, 239-246.

[32] Sanders C. L., Dagle G. E., Cannon W. C., Craig D. K., Powers G. J., MeIER D. M. Inhalation cancerogenesis of high fired ${ }^{239} \mathrm{PuO}_{2}$ in rats. Radiat. Res., 1976, 68, 349-360.

[33] Masse R., Ducousso R., Nolibe D., Lafuma J., Chrétien J. Passage transbronchique des particules métalliques. Rev. Fr. Mal. Resp., 1974, 2, 123-127.

[34] Sanders C. L., Powers G. J. Cocarcinogenesis of inhaled plutonium dioxide and beryllium oxide. Pacific Northwest Laboratory annual report 1976, BNWL-2100, PT 1, 1977, 33-35.

[35] Fritsch P., Masse R., Stanislas G., Chrétien J. Étude dynamique comparative de la population cellulaire intra-alvéolaire après injection intratrachéale d'hydroxyde de beryllium et d'hydroxyde d'aluminium chez le rat. Biomédecine, 1975, 23, $\mathrm{n}^{\circ}$ 3, 97-102.

[36] LyubChanskiı E. R. The behavior of Pu-239 in rats after a single inhalation of some of its chemical compounds. Radiobiology [U.S.S.R. (English tr.)], 1967, 7, n ${ }^{\circ} 4,84-96$. 
[37] Nenot J. C., Masse R., Morin M., Lafuma J. An experimental comparative study of the behaviour of ${ }^{237} \mathrm{~Np},{ }^{238} \mathrm{Pu},{ }^{239} \mathrm{Pu},{ }^{241} \mathrm{Am}$ and ${ }^{242} \mathrm{Cm}$ in bone. Health Phys., 1972, 22, 657-665.

[38] McClellan R. O., Boyds H. A., Gallegos A. F., Thomas R. G. Retention and distribution of ${ }^{244} \mathrm{Cm}$ following inhalation of ${ }^{244} \mathrm{CmCl}_{3}$ and ${ }^{244} \mathrm{CmO}_{1.73}$ by beagle dogs. Health Phys., 1972, 22, 877-885.

[39] Thomas R. G., McClellan R. O., Thomas R. L., Chiffelle T. L., Hobbs C. H., Jones R. K., Mauderly J. L., Pickrell J. A. Metabolism dosimetry and biological effects of inhaled ${ }^{241} \mathrm{Am}$ in beagle dogs. Health Phys., 1972, 22, 863-871.

[40] Mahaffey J. A., Merrill J. A., Sanders C. L. Metabolic modeling of inhaled ${ }^{244} \mathrm{CmO}_{2}$. Pacific Northwest Laboratory annual report 1977, BNL-2500 PT1, 1978, 344-345.

[41] Lafuma J., Nenot J. C., Morin M., Masse R., Métivier H., Nolibe D., Skupinski W. Respiratory carcinogenesis in rats after inhalation of radioactive aerosols of actinides and lanthanides in various physicochemical forms. In : Experimental Lung Cancer (Karbe E., Park J. F., éd.). Berlin, Springer Verlag, 1974, 1, 443-453.

[42] MASSE R. Communication personnelle.

[43] Воососк G., Popplewell D. S. Distribution of plutonium in serum proteins following intravenous injection into rats. Nature, Londres, 1965, 208, 282-283.

[44] Popplewell D. S., Boocock G. Distribution of some actinides in blood serum proteins. In : Diagnosis and Treatment of Deposited Radionuclides, Richland, May 15-17, 1967. (Kornberg H. A., Norwood W. D., Eds.). Amsterdam, Excerpta Medica Foundation, 1968, 44-45.

[45] Stradling G. N., Popplewell D. S., Ham G. J. The chemical form of americium and curium in urine. Health Phys., 1976, 31, 517-19.

[46] Masse R., Métivier H., Guillaumont R. Fixation osseuse des terres rares et des éléments transuraniens. In : Physicochimie et cristallographie des apatites d'intérêt biologique, Paris, 10-15 septembre 1973, Colloques Internationaux C.N.R.S. $\mathrm{n}^{\circ} 230$, Paris, C.N.R.S. 1974, 441-450.

[47] Masse R., Nolibe D., Fritsch P., Métivier H., Lafuma J., Chrétien J. Chronic interstitial pneumonitis induced by internal $\alpha$-irradiation of the lung. In : Alveolar interstitium of the lung. Prog. Resp. Res., 1975, 8, 74-90.

[48] PARK J. F., BaIR W. J., BusCh R. H. Progress in beagle dogs studies with transuraniun elements at Battelle-Northwest, Health Phys., 1972, 22, 803-810.

[49] Métivier H., Nolibe R., Masse R., Lafuma J. New data on toxicity and translocation of inhaled ${ }^{239} \mathrm{PuO}_{2}$ in baboons. Health Phys., 1978 (sous presse).

[50] Lustgarten C. S., Boecker B. B., Cuddihy R. G., Kanapilly G. M. Biliary excretion of ${ }^{144} \mathrm{Ce}$ after inhalation of ${ }^{144} \mathrm{Ce}$ citrate in rats and syriam hamsters. Inhalation Toxicology Research Institute annual report 1975-1976, LF-56, 1976, 84-87.

[51] Durbin P. W. Metabolism and biological effects of the transplutonium elements. In : Uranium, plutonium, transplutonic elements (Hodge J. C., StanNaRd J. N., HuRsh J. B., Eds.). Berlin, Springer Verlag, 1973, 739-896.

[52] PARK J. F. Communication personnelle.

[53] Park J. F., Lund J. E., Ragan H. A., Hackett P. L., Frazier M. E. Bone tumors induced by inhalation of ${ }^{238} \mathrm{PuO}_{2}$ in dogs. In : Recent results in cancer research. Berlin, Springer Verlag, 1976, 54, 17-35.

[54] Craig D. K., Decker J. R., Powers G. J., Parf J. F. Disposition of highly toxic radioactive aerosols inhaled by beagle dogs. Annual meeting of the Health Physics Society, Buffalo, New York, July 1975.

VoL. $14-\mathrm{N}^{\circ} 1$ 
[55] Nolibe D. Étude de la rétention de l'oxyde de plutonium inhalé. Modalités de son élimination par lavage pulmonaire et rôle du macrophage alvéolaire, Thèse, Paris 1976. Rapport CEA-R-4830.

[56] Bair W. J., Tombropoulos E. G., PARK J. F. Distribution and removal of transuranic elements and cerium deposited by the inhalation route. In : Diagnosis and treatment of radioactive poisoning, Vienne, A.I.E.A. 1963, 319-338.

[57] Lincoln T. A. The use of DTPA and induced sputum as diagnostic techniques after internal contamination. In : Diagnosis and treatment of incorporated radionuclides, Vienne, 8-12 décembre 1975. Vienne, I.A.E.A. 1976, 203-208.

[58] Nolibe D. Élimination par lavage pulmonaire, in vivo, des particules d'oxyde de plutonium inhalées. II. Choix de la période d'intervention. C. R. Acad. Sc., Paris, série D, 1973, 276, 681-684.

[59] Nolibe D. Élimination par lavage pulmonaire in vivo, des particules d'oxyde de plutonium inhalées. I. Description de la technique utilisée. C. R. Acad. Sc., Paris, série D, 1973, 276, 225-228.

[60] Nolibe D., Nenot J. C., Metivier H., Masse R., Lafuma J. Traitement des inhalations accidentelles d'oxyde de plutonium par lavage pulmonaire, in vivo. In : Diagnosis and treatment of incorporated radionuclides, Vienne, 8-12 décembre 1975. Vienne, I.A.E.A. 1976, 373-385.

[61] Nolibe D., Métivier H., Masse R., LAfuma J. Therapeutic effect of pulmonary lavage, in vivo, after inhalation of insoluble radioactive particles. In : Inhaled particles IV (Walton W. H., Ed.). Oxford, Pergamon Press, 1977, 2, 597-613.

[62] Nolibe D. Communication personnelle.

[63] Richmond C. R. The importance of non uniform dose distribution in an organ. Health Phys., 1975, 29, 525-537.

[64] Miglio J. J., Pritt J. W. Solubility characteristics of some laboratory produced mixed oxide aerosols. Inhalation Toxicology Research Institute annual report 1975-1976, LF-56, 1976, 25-27.

[65] Brightwell J., Carter R. F. Comparative measurements of the short-term lung clearance and translocation of $\mathrm{PuO}_{2}$ and mixed $\mathrm{Na}_{2} \mathrm{O}+\mathrm{PuO}_{2}$ aerosols in mice. In : Inhaled particles IV (WALtoN W. H., Ed.). Oxford, Pergamon Press, 1977, 2, 285-302.

[66] Stather J. W., Howden S., Carter R. F. A method for investigating the metabolism of the transportable fraction of plutonium aeorosols. Phys. Med. Biol., 1975, 20, 106-124.

[67] Stradling G. N., Loveless B. W., Ham G. J., Smith H. The biological solubility in the rat of plutonium present in mixed plutonium sodium aerosols. Health Phys., 1978 (sous presse).

[68] Brightwell J., Carter R. F. Absorption from the gut of a suspension of plutonium sodium aerosol. Health Phys., 1975, 28, 156-158.

[69] Smith H., Stradling G. N., Loveless B. W., Ham G. J. The in vivo solubility of plutonium 239 dioxide in the rat lung. Health Physics, 1977, 33, 539-551.

[70] Métivier H., Masse R., Rateau G., Nolibe D., Lafuma J. Experimental study of respiration contamination by a mixed oxide aerosol formed from the combustion of plutonium magnesium alloy. Health Phys. (à paraître).

[71] Métivier H., Masse R., Nenot J. C., Nolibe D., Lafuma J. Étude expérimentale de la contamination par un mélange issue de la combustion du sodium en présence d'oxyde de plutonium. In : Diagnosis and treatment of incorporated radionuclides, Vienne, 8-12 décembre 1975. Vienne, A.I.E.A. 1976, 107-117. 
[72] Métivier H., Masse R., Nolibe D., Nenot J. C., Lafuma J. Effect of ascorbate ions in DTPA treatment after contamination by mixed plutonium dioxide-sodium burning compounds. Health Phys., 1977, 32, $\mathrm{n}^{\circ}$ 5, 450-452.

[73] Ducousso R., Causse A., Masse R., Kermarec J. Bronchographies par aérosol de tantale : étude toxicologique de l'opacifiant, J. Radiol. Electrol. 1973, 54, n' ${ }^{\circ} 11,813-821$.

[74] Bianco A., Gibb F. R., Kilpper M. S. R. W., Landman S., Morrow P. E. Studies of tantalum dust in the lungs, Radiology, 1974, 112, 549-556.

[75] Gore D. J., Thorne M. C. The distribution and clearance of inhaled uranium dioxide particles in the respiratory tract of the rat. In : Inhaled particles IV (WALTON W. H., Ed.). Oxford, Pergamon Press, 1977, 1, 275-283.

[76] Masse R., Fritsch P., Ducousso R., Lafuma J., Chrétien J. Rétention de particules dans les cellules bronchiques, relations possibles avec les carcinogènes inhalés. C. R. Acad. Sc., Paris, série D, 1973, 276, 2923-2925.

[77] Radford E. P., Martell E. A. Polonium 210 : lead 210 ratios as an index of residence times of insoluble particles from cigarette smoke in bronchial epithelium. In : Inhaled particles IV (Walton W. H., Ed.). Oxford, Pergamon Press, 1977, 2, 567-581. 